\title{
Gender Dysphoria and Transgender Identity Is Associated with Physiological and Psychological Masculinization: a Theoretical Integration of Findings, Supported by Systematic Reviews
}

\author{
Edward Dutton ${ }^{1} \cdot$ Guy Madison ${ }^{2}$ (1) \\ Published online: 13 August 2020 \\ (C) The Author(s) 2020
}

\begin{abstract}
Introduction Gender dysphoria (GD) is associated with several psychiatric conditions, but the causal links are not known. We note that some of these conditions are associated with physiological masculinisation.

Methods Here, we explore this association through a series of systematic reviews, using Google Scholar, on original studies that test the relationship between GD and at least one correlate of androgens, namely autism spectrum disorder, left-handedness, 2D:4D ratio, being male and male heterosexuality.

Results Individuals with GD tend to exhibit scores that reflect heightened levels of androgens and masculinity compared with non-GD individuals. We further show that these same androgen indices are also associated with other identity disorders (or dysphoriae).

Conclusions Autism is associated with masculinisation, and we argue that GD may reflect autism spectrum disorder traits that indirectly lead to anxiety and to one questioning one's sense of self. We note that this is consistent with Blanchard's transsexualism typology, which successfully integrates a wide range of empirical findings.
\end{abstract}

Keywords Gender dysphoria · Masculinisation · Transsexuality · Autism spectrum disorder · Androgens $\cdot$ Identity

\section{Introduction}

Gender dysphoria (GD) refers to a state whereby a person is dissatisfied with the sex assigned to them at birth and the gender role that it entails. They may, as a consequence, desire sex reassignment (Fraser, 2015, p. 19). The DSM-5 defines GD as 'an individual's affective/cognitive discontent with the assigned gender', adding that it may also refer to 'distress that may accompany the incongruence between one's experienced or expressed gender and one's assigned gender' (American Psychiatric Association, 2013). GD was previously known as Gender Identity Disorder in the DSM-III, though this only referred to child diagnoses (American Psychiatric Association, 1980). The exact causes of GD remain a subject

Guy Madison

guy.madison@umu.se

Edward Dutton

ecdutton@hotmail.com

1 Ulster Institute for Social Research, 118 Purves Road, London, UK

2 Department of Psychology, Umeå University, Umeå, Sweden of debate and a widely propounded model, which we will explore below, is that the condition relates to in utero oestrogen exposure. However, as we will explore below, research in quite separate disciplines has suggested that GD is associated with early androgen exposure (Leinung \& Wu, 2017) which would seem to contradict the female hormone explanation. Here, we will attempt to bring these diverse findings together into an overarching model which makes sense of the relationship between masculinisation and GD. First, we provide a review of the associations between GD and androgen exposure and of possible causal explanations for GD. Based on this, we select a set of indicators known to be associated with androgens and masculinisation. Then, we perform a series of systematic reviews on each of these indicators and GD, the results of which are compared with the predictions implied by the different explanations for GD.

In terms of understanding the psychological mechanisms behind this putative association between masculinisation and GD, we will argue below that autism spectrum disorder (ASD) may be highly relevant. ASD refers to 'persistent deficits in social communication and social interaction', especially with regard to empathy, combined with restrictive and repetitive patterns of behaviour, interests or activities (American 
Psychiatric Association, 2013). We note that autism and ASD are associated with several physiological and psychological indicators of masculinisation, such as higher levels of androgens (Ruta et al., 2011), even in early childhood (Auyeung et al., 2010), being left-handed and having a lower digit ratio (defined below). There are a number of theories that attempt to explain why this is the case. One of the most influential ones is Baron-Cohen's (2002) 'Extreme Male Brain Theory of Autism'. This theory attempts to describe different cognitive profiles in the general population, with a focus on explaining ASD. As such, it brings together a substantial body of evidence for systematic differences in males' and females' psychological functioning, even in very early childhood. Covering both typical development and functioning, as well as several forms on non-typicality, it can potentially be useful for theoretically integrating our observations. According to Baron-Cohen, the stereotypical 'male brain' is focused on 'systematising': analysing the variables in a system, working out its rules and developing systems to make sense of new situations. The 'female brain' is instead more geared towards 'empathising': identifying other persons' emotions and thoughts and working out the best way to respond to them. Baron-Cohen reviews evidence that girls and women are superior at, or more interested in, empathising. Compared with males, females have higher verbal ability, are better at turntaking, less interested in physically rough games (as they may injure people), are more likely to comfort strangers and share the distress of their friends to a greater extent than males, have a better theory of mind than males, value harmonious relationships over power-centred ones and are less aggressive. Compared with females, males prefer building-related toys, occupations focused on mechanical or theory-based systems (such as construction, engineering, computer-programming, or auto mechanics) and math-based subjects (such as theoretical physics). They have superior mechanical reasoning abilities, better spatial ability, a finer eye for detail, better mapreading skills, superior motor skills (making them better at hitting a target), greater ability and interest in organising and classifying and are less prone to allow extraneous information to interfere with these processes. Baron-Cohen argues that the most extreme male brain would, therefore, be autistic: very low in empathy but very high in systemising. The extreme female brain, by contrast, would be highly empathetic and emotionally driven, but would be 'system blind' or prone to developing empirically inaccurate models of reality. A recent study, drawing upon 658,000 people, confirmed that there are significant sex differences on the autism spectrum in line with Baron-Cohen's theory and that autistics exhibit a particularly masculinised physical and psychological profile (Greenberg et al., 2018).

Baron-Cohen acknowledges potential cultural influences in the observed sex differences, but the evidence he presents indicates significant biological causes. For example, they are present early in ontogenesis, exhibit the same patterns crossculturally, are associated with physiological factors that are unlikely to be environmentally influenced, such as hormones, and are consistent with evolutionary theory (e.g. Geary, 2017). For reviews, see Bale and Epperson (2017), Berenbaum and Beltz (2016), Cahill (2006), Hausmann (2017), Lippa (2010) and Su et al. (2009). Baron-Cohen (2002) observes that those who score high on the autism spectrum are not merely masculinised in how they think but also on certain key physiological markers, though it should be noted that the evidence is mixed. According to BaronCohen, autistic males have high circulating testosterone levels, expressed in precocious puberty, and a lower 2D:4D digit ratio, which has been associated with high prenatal testosterone levels (Manning et al., 2001). 2D:4D is the ratio of the index finger to the ring finger, which are the two fingers that seem to provide the most sensitive measure of individual differences in prenatal androgen exposure (Manning et al., 2001). The 2D:4D ratio is a relatively weak indicator generally, both because of measurement problems (e.g., Ribeiro et al., 2016) and because of large individual differences (e.g., Swift-Gallant et al., 2020). Still 2D:4D is, on average, lower in males according to a meta-analysis (Hönekopp \& Watson, 2010) and a large cross-cultural sample with more than 160,000 participants (Manning et al., 2017), which also showed highly significant correlations between 2D:4D and several indices of femininity/masculinity. Some studies have found that testosterone exposure does not reduce empathy in males (Nadler et al., 2019) or that autistic men are not more likely to be psychologically masculine or heterosexual (Bejerot \& Eriksson, 2014), though these are based on relatively small samples and are contradicted by yet other studies. For example, 'tomboy' identity among girls is associated with higher levels of prenatal testosterone (Atkinson et al., 2017). This would imply that the girls are masculinised, although not necessarily suffering from GD. Thus, such findings are tangential evidence for the masculinisation thesis.

Autism is also associated with being left-handed, with lefthandedness being another marker of masculinisation, being around 2 percentage points more common in males (Papadatou-Pastou et al., 2008). One of few studies on handedness in autistic children found that $62 \%$ of the children were left-handed, compared with $37 \%$ of controls matched for various criteria (Gillberg, 1983), in agreement with a positive association between autism and left-handedness (e.g. Kobylynska et al., 2017). A meta-analysis of 144 studies found that males are more likely than females to be left-handed, consistent with the correlation between autism and masculine physical traits (Papadatou-Pastou et al., 2008). Accordingly, left-handed people have higher levels of serum testosterone (Sperling et al., 2010), and homosexual females tend to be masculinised both physically and in terms of personality and cognitive profile - though there is much variation 
within this category (for a review, see Balthazart, 2011). Congruous with this finding, $45 \%$ of self-identified feminists in a US sample identified as non-heterosexual and predominantly homosexual and a number of studies have found that homosexual women are substantially more likely to be lefthanded than are heterosexual women. This difference is also found between homosexual and heterosexual males, although to a much smaller extent (Mustanski et al., 2002; Blanchard \& Lippa, 2007; Ellis et al., 2017), as also shown by a metaanalysis (Lalumiere et al., 2000). It should be noted that all the associations reviewed above constitute the dominating pattern, while not each and every reviewed study finds the relationships in question. That will, naturally, be true of any literature review.

Having briefly reviewed Baron-Cohen's model and the empirical support for the relation between physical masculinisation correlates of ASD that it entails, we will now consider its weaknesses. Baron-Cohen's model has been criticised on the grounds that it describes, rather than explains, sex differences in cognition, until physical evidence is presented to indicate that female brains differ from male brains (Ridley, 2019). However, Ridley does not acknowledge much of the evidence that already exists. Female brains are on average smaller than male brains, even when controlling for body size, and have less grey and white matter than males (Burgaleta et al., 2012, Table 1; van der Linden et al., 2017). For reviews of structural and functional sex differences in the brain, see, e.g. Bale and Epperson (2017), Hausmann (2017), Gur and Gur (2017), Hirnstein et al. (2019), Jahanshad and Thompson (2017) and Lai et al. (2017). Moreover, children with diagnosed autism-defined as the extreme male brain - display neurological differences from controls, meaning that it cannot be argued that BaronCohen's model somehow works for female autistics but not for male autistics. It is rather the case that neurologically problematic conditions are more common among males (Sparks et al., 2002).

\section{Gender Dysphoria and Key Hypotheses}

With Baron-Cohen's model and its correlates clear, let us now consider alternative explanations for gender dysphoria (GD). A person whose gender identity is incongruous with their biological sex may be diagnosed with GD if that person experiences persistent discomfort with their sex or the gender role it entails. A person whose gender identity does not correspond with their physiological sex or assigned gender is transgender, and those who undergo surgery or hormone therapy to change their physical sexual characteristics are transsexual (Parekh, 2016). There is also the important distinction between early-onset and adolescent-onset GD, which manifest in early childhood or after the onset of puberty, respectively. As we will see later, early GD is much more strongly associated with having physical and mental markers of the opposite sex (Zucker et al., 2016). We will use the term GD to encompass the general phenomenon of being uncomfortable with one's assigned or physiological sex, which is central for the present thesis. We will use the term transgender when we refer to groups who are transgender or transsexual, assuming they experience or have experienced GD.

The most generally accepted explanation for GD seems to be developmental instability in utero, combined with genetic factors. Developmental instability refers to an individual's inability to produce a specific phenotype under environmental conditions, though this may be partly mediated by their genetics (Pertoldi et al., 2006). We will call this the 'genetic/hormonal model'. This model is based on the existence of modal brain differences between transgender persons and so-called $c i s$-gendered individuals and that the brains of transgender persons have features more similar to those of the gender with which they identify than those of their biological sex. (e.g. Luders et al., 2009). It has further been proposed that this may be caused by the pregnant mother producing too little or too much oestrogen (e.g. Luders et al., 2009) combined with the child having the long form of the receptor allele for testosterone or androgen (e.g. Hare et al., 2009). While this

Table 1 Gender dysphoria and 2D:4D ratio

\begin{tabular}{lcc}
\hline Study & Sample & Finding \\
\hline Leinung and Wu (2017) & 118 transgender subjects & Subjects have lower 2D:4D than controls \\
Wallien et al. (2008) & 1. 96 GD males and 51 GD females compared with & 1. Females with GD had lower 2D:4D ratio \\
& 2. 67 hD boys and 34 GD girls compared to 74 control & 2. No difference. \\
& boys and 72 control girls. & Right hands of right-handed transmen \\
hraemer et al. (2009) & 56 GD patients (39 transwomen and 17 transmen) & Male GD lower 2D:4D in both hands; \\
& with control sample of 176 men and 190 women & female GD lower in right hand. \\
Hisasue et al. (2012) & 20 male and 20 female medical students measured & Lower 2D:4D than controls.
\end{tabular}


developmental instability model may capture some instances of GD cases, it suffers from a number of problems at the general level meaning that there is likely a more nuanced alternative explanation.

Firstly, meta-analyses of twin studies indicate that the heritability of GD is around 0.5 and perhaps considerably less (Polderman et al., 2018). Thus, such an explanation cannot fully explain the patterns of GD we observed above. Secondly, although the genetic/hormonal model might explain early-onset GD in terms of, for example, a male being born with an essentially female brain, it does not explicitly account for the phenomenon of adolescent-onset GD, because evidence of primarily genetic/hormonal issue should manifest early. It is especially problematic when it comes to the recent surge in early and mid-adolescents very suddenly, and with no previous symptoms of GD, declaring to be non-binary or identifying as a member of the opposite sex, which has been termed rapid-onset GD and seems to spread rapidly through female social groups via social contagion. It has been noted that this should be distinguished from adolescent GD, due to its different correlates and mechanisms (Littman, 2018). The genetic/hormonal model would, thirdly, assume a corresponding change in the foetal milieu of mothers in recent decades, for which no evidence has been recorded. Littman suggests that rapid-onset GD is an expression of a 'social coping mechanism' for other issues, such as adolescent homosexual phases, rendered increasingly common by social contagion. This would be far more consistent with a primarily environmental explanation, at least for adolescent-onset GD. Fourthly, it has been argued that hormonal imbalances in utero are associated with homosexuality, creating masculinised females or feminised males (Balthazart, 2011). This raises the question of what factors specifically lead to transsexuality rather than homosexuality. Environmental variables seem a reasonable possibility, and, if so, a detailed environmental model should be developed. Fifthly, a primarily environmental explanation would be consistent with a growing body of evidence of transsexual persons who wish to revert back such that they physically resemble their biological sex (e.g. Turban \& Keuroghlian, 2018; Danker et al., 2018). Sixthly, the common explanation for transsexuality does not explain why there should be an association between GD and being sexually aroused by cross-dressing, as reported by $80 \%$ of a sample of transwomen (male-to-female) (Blanchard et al., 1987). Some research has found that these childhood 'gender conflicts' are true of future transmen (female-to-male) as well (Houssayni \& Nilsen, 2018). Finally, it fails to explain evidence of a relationship between GD and other dysphoria, such as anorexia (Ristori et al., 2019).

In contrast, we contend that GD may, in part, be a manifestation of masculinisation. If supported by the following review of the empirical data, we will develop the reasons for this contention in the 'Discussion'. If GD is a manifestation of masculinisation, then we hypothesise that those experiencing GD exhibit: (1) elevated levels of ASD traits, (2) elevated levels of left-handedness and (3) a lower 2D:4D ratio than the typical person of their assigned sex. We make these three predictions because all these traits are predicted by testosterone or other androgens. We also hypothesise that (4) gender reassignment surgery or clinical GD is far more common in the direction male-to-female (transwoman) than in the direction female-to-male (transman), because natal males have higher levels of androgens and are more masculinised. Similarly, (5) transwomen should exhibit an elevated level of attraction to females relative to the level of their non-natal sex. We make these predictions because males' higher levels of androgens should be associated with ASD traits and because male homosexuality is associated with being feminised and thus being lower in male hormone levels. In addition, we hypothesise that (6) these predictions also apply to individuals who experience other forms of dysphoria. We make this prediction because we have already observed that different dysphoriae correlate with each other and because GD should therefore resemble other dysphoriae in its correlates. In this first exploration, these predictions will be analysed with respect to secondary data based on published research literature, as described in our 'Methods and Results' section.

The alternative genetic/hormonal explanation, based on foetal oestrogen levels and genetics, would predict (1) no association between GD and autism traits, (2) that there might be an association between GD and left-handedness, due to this also being a manifestation of developmental instability (Woodley et al., 2018) represented by elevated or reduced in utero hormones and (3) that transwomen would have a higher 2D:4D than cis-males. Furthermore, it would predict (4) no significant sex differences in the direction of gender reassignment surgery, and that (5) transgender person groups would have the same proportion of homosexuality as cis-gendered groups. The following systematic reviews therefore include any study designs that enable the following comparisons, focus on transgender persons, while for some questions also including homosexual persons and persons with other dysphoriae, consider associations but not interventions, and compare the sexes and the variables and conditions implicated in the review above, namely autism spectrum disorder, lefthandedness, 2D:4D ratio and sexual orientation.

\section{Methods and Results}

\section{ASD and Gender Dysphoria}

A systematic review of the ASD-Gender Dysphoria nexus was recently conducted, and we did therefore not need to conduct one ourselves. This review found a robust relationship between ASD and GD (van der Miesen et al., 2016). 
While it has received criticism for using symptomatology scales that 'might not be specific to ASD' (Turban \& Van Schalkwyk, 2018), the authors have responded (Van der Miesen et al., 2018) that many studies that employ stricter ASD scales still find an association. Since that review was conducted, Heylens et al. (2018) found that transgender persons were six times more likely than the general population to suffer from ASD, with transwomen (male-to female) being more likely to have an ASD than transmen (female-to-male). van der Miesen et al. (2018) found that children with GD score higher on all subdomains of ASD than do controls. Likewise, the systematic review by Glidden et al. (2016) found that people with GD have a higher rate of ASD than the general population, based on 19 studies between 1966 and 2015 . Indeed, there have since been other reviews confirming the relationship between ASD and GD (e.g. Øien et al., 2018) and a further original empirical study among those who self-define as 'transgender' or 'non-binary' (Stagg \& Vincent, 2019). One review (Dewinter et al., 2013) insists there is no clear relationship between autism and sexual identity issues, but this appears to be due to extremely tight inclusion criteria, such as refusing to include studies which employ behaviour or testimony from parents. Moreover, it explores normative sexual development more generally rather than specifically GD (Dewinter et al., 2013). Thus, we conclude that GD is robustly associated with ASD.

\section{Gender Dysphoria and Left-Handedness}

Next, we explored the GD and Left-handedness nexus. The rate of left-handedness among the general population in Western countries is about $12 \%$ (Woodley et al., 2018). We could find no systematic review on the relationship between left-handedness and GD. We therefore conducted one in March 2019, following the PRISMA guidelines (http:// www.prisma-statement.org/). The inclusion criteria were that the study was original and tested the relationship between these two variables. Based on these criteria we searched Google Scholar, which is a particularly useful database by virtue of including both scientific articles and academic theses. We first searched for 'handed' AND 'transgender'. This produced 600 hits of which only Jones et al. (2012) appeared potentially relevant. The other hits did either not actually test any of the associations or did not report original data and were therefore ignored. Jones et al. (2012) cited three studies of direct relevance: Green and Young (2001), Orlebeke et al. (1992) and Soper et al. (1986). We repeated the search with handed and transsexual. This gave us 5430 hits, of which the only new and relevant one was Watson and Coren (1992). A search with handed AND 'gender identity disorder' gave 1030 hits, of which Zucker et al. (2001) was within our inclusion criteria. We repeated all these searches replacing handed with 'sinistrality', but this did not reveal anything new. In the next step of the PRISMA procedure (Data Management), all titles were copied into a word document. Finally, the selection process was conducted. Based on the general criteria outlined above and on further analyses of the content of the articles (e.g. title, abstract, text), it was decided to include Jones et al. (2012), Green and Young (2001), Orlebeke et al. (1992), Soper et al. (1986), Watson and Coren (1992) and Zucker et al. (2001).

All of these studies found elevated left-handedness among those with GD, including both transmen and transwomen. In alphabetical order, Green and Young (2001) found that transmen exhibit elevated levels of non-right-handedness, Watson and Coren (1992) found that 35\% of male-to-female transgender persons were left-handed, as compared with $11.6 \%$ in a control sample, and Zucker et al. (2001) found that $19.5 \%$ of boys with GD were left-handed compared with $11 \%$ among controls but did not find a significant difference for girls. Watson and Coren argue that this is consistent with the strongly elevated level of left-handedness among masculinised females, proposing that left-handedness may be an effect of various early stressors, such as preterm birth, breach birth and multiple birth, because these stressors elevate the production of testosterone. We note that this mechanism is directly relevant to our model. Watson and Coren (1992) further argue that autism is itself associated with preterm birth and left-handedness, which are in turn commonly accepted (see Baron-Cohen, 2002) to be associated with testosterone (Committee on Understanding Premature Birth, 2007).

\section{Gender Dysphoria and 2D:4D Ratio}

Voracek et al. (2018) recently conducted a meta-analysis of the relationship between 2D:4D ratio and transgender identity and found that there was a small but significant relationship in the direction that we predicted. However, this was freshly published when we commenced this study and it did not come to our attention. So, in March 2019, we conducted a systematic literature review, again following the PRISMA guidelines as set out above. Our inclusion criteria were that the study was original and tested the relationship between the two variables. We first searched Google Scholar using the terms 'trans*' AND '2D:4D'. This revealed 470 studies of which Leinung and $\mathrm{Wu}$ (2017) and Wallien et al. (2008) were within the inclusion criteria. This search also turned up studies with the words 'digit ratio' and 'finger ratio', so we did not need to separately search for these. We then searched for 2D:4D AND gender identity disorder. This gave 100 hits, of which seven were relevant and two that had not been found by the previous search (Kraemer et al., 2009; Hisasue et al., 2012). We repeated the search once more using 2D:4D AND 'gender dysphoria'. This gave us 100 results of which one was both new and relevant (Vujović et al., 2014). We also checked the reference lists of articles within our inclusion criteria, but no further 
germane studies were revealed. The results of this literature review are listed in Table 1. It turned out that, when we compared this result, based on our own keywords and inclusion criteria, we found exactly the same studies as did Voracek et al. (2018).

As can be seen from Table 1, only Kraemer et al. (2009) is not in line with our predictions, but it used only the left hand, which is likely to make the results invalid for the present purpose. This is because 2D:4D is itself associated with handedness, with the left-handed tending to have a higher 2D:4D ratio (e.g. Datta Banik, 2017). Because transgender persons are more often lefthanded (Blanchard \& Lippa, 2007), this may lead to an overestimation of the effect of GD on left-handedness, especially as the samples involved are relatively small. Otherwise, the results are partly or wholly as predicted, and no study, except Kraemer et al.'s (2009), contradicts the hypothesis.

\section{Male-to-Female Transgender Is More Prevalent}

To reiterate, GD prevalence should be higher among biological males than among biological females if it is related to masculinisation. The US Social Security Administration has made an attempt to estimate the proportion of transmen and transwomen among transgender persons among a large sample of 135,367 likely transgender persons based on registered name changes between the years 2002 and 2013. Of these, $65 \%$ involved a change from a male to a female Christian name while it was the other way around with the remaining $35 \%$. Somewhere between 0.1 and $0.3 \%$ of the US population are estimated to be transgender (Miller, 2015). The higher proportion of transwomen is consistent with our hypothesis. Based on smaller samples, a recent study of children in Finland aged between 16 and 18 (N 1746) found that 'clinically significant GD was $3.6 \%$ among males and $2.3 \%$ among females' (Kaltiala-Heino et al., 2019, Abstract). In addition, a systematic literature review found that fewer than ' 1 in 10,000 adult natal males and 1 in 30,000 adult natal females experience GD' (Zucker et al., 2016, Abstract). Goodman et al. (2019) have recently attempted to estimate the size of transgender populations and aver that percentages of female-tomale transgender persons have increased over time. It should be emphasised that, as we will discuss in greater detail below, environmental factors may substantially influence gender identity. A rapid increase in only a few decades is therefore not surprising, in light of profound societal and the attitudinal changes. Notably, such an increase does not undermine the influence of heritable factors on the propensity for GD.

\section{Transgenderism and Homosexuality}

Male homosexuality is associated with feminisation (Miller, 2000) whereas lesbianism is associated with masculinisation (Jeffrey et al., 2019). Inasmuch as transwomen have GD, they should also be masculinised, according to our hypothesis, and therefore be mainly attracted to females. In the UK in 2016, $2 \%$ of the population over the age of 16 claimed to be lesbian, gay or bisexual. Among the younger cohort of 16 to 24 year olds, it has doubled to $4.1 \%, 2.3 \%$ of whom are gay (male homosexual), $1.6 \%$ lesbian and $0.2 \%$ bisexual (Office of National Statistics, 2016). To assess the sexual orientation of transgender persons requires very large surveys, because of their rarity. A survey of 3000 transwomen conducted in 2015 reported that at least $60 \%$ claimed to be gynephilious (sexually attracted to women), $27 \%$ were exclusively gynephilious and $19 \%$ exclusively androphilious. Among transmen, in contrast, only $12 \%$ were exclusively gynephilious while $23 \%$ were exclusively androphilious, but the proportions of those who were non-exclusively attracted to either sex were not reported (James et al., 2016). Thus, homosexuality with respect to the acquired gender is strongly elevated among transwomen in comparison with transmen. This is in line with the hypothesis, congruous with transgender persons being masculinised. It should be noted that among early-onset GD (which is found in children), the individuals are generally attracted, in adulthood, to their own natal sex. By contrast, adolescent-onset GD persons are to a greater extent attracted to the opposite of their natal sex (Zucker et al., 2016). This implies that early-onset GD is more consistent with 'being born into the wrong body' than is adolescent-onset GD.

\section{Other Dysphoria and Extreme Male Brain Indicators}

If gender dysphoria were strongly related to a masculinised brain, then this state should also be associated with other forms of dysphoria. This is because we would expect all dysphoria to have similar correlates insomuch as they are all, to a certain extent, the fundamental issue. Anorexia is a prominent condition in which anorexic persons believe themselves to be overweight when in actuality being thin and often dangerously undernourished. If this is related to GD or its correlates, this is unlikely to be militated by other factors because both conditions involve the same essential condition: a superficially empirically inaccurate self-image. In other words, they can be argued to be, broadly, the same kind of condition. A relevant observation is that anorexic girls are 2.8 times more likely to be left-handed than are healthy controls (Tenconi et al., 2010). Likewise, Quinton et al. (2011) found that the 2D:4D ratio is significantly lower - that is, more masculinised-among females with anorexia than among healthy controls. There has already been a systematic literature review on the relationship between anorexia and ASD, which demonstrated a clear association across eight studies (Westwood \& Tchanturia, 2017). Anorexia is, however, far more common among females than males, but this is true of all eating disorders, including Bulimia, a disorder which is positively correlated with 2D:4D ratio. The likely reason for this is that females are 
fundamentally more prone to develop psychiatric conditions related to their physical attractiveness, due to heritable influences based on sexual selection, as attractiveness it is substantially more important for fertility and number of offspring than it is for males (see Buss, 1989). The critical result is, however, that females with body dysphoria exhibit signs of physiological masculinisation, consistent with the hypothesis. Thus, physiological masculinisation is associated with dysphoria, although other factors - such as a female focus on the importance of being physically attractive - may influence the precise nature of the dysphoria. Accordingly, there is no contradiction between anorexia being a primarily female condition and it concomitantly being a manifestation of masculinisation associated with ASD.

Another, far rarer, dysphoria is body integrity disorder, in which a person is obsessed with a desire to amputate a healthy limb. This condition is so unusual that there is relatively little research into its correlates. However, in line with our hypothesis, it is predominantly a male condition. In a systematic review of 14 studies on this topic, 11 only involved males. According to that review, one study indicates that $94 \%$ of those who present with this disorder are male. It is also associated with a history of poor social relationships, with gender dysphoria and with depression (Khahil \& Richa, 2012).

\section{Discussion}

We compared two models that attempt to account for GD, the prevalent genetic/hormonal explanation and our proposed masculinisation model. It can be seen that all of our six hypotheses were supported by the research literature. People with GD have elevated levels of (1) ASD traits and (2) lefthandedness and (3) a lower 2D:4D ratio, (4) gender reassignment surgery or clinical GD is more common in transwomen than transmen, (5) transwomen are gynephilious more often than transmen are androphilious and (6) these predictions apply also to individuals who experience other forms of dysphoria.

Four of the predictions from the alternative genetic/ hormonal hypothesis are different from those of the masculinisation model, and so were all contradicted. The exception is the hypothesis regarding left-handedness, which is the same for both models, indicating support for both. However, the evidence was not so strong, partly because of being confounded with handedness, measuring only the left hand. Our approach has been to form a pattern of predictions, of which any violation would render the model less tenable, in line with the aims of consilience (Wilson, 1998). Taken together, we therefore argue that the results provide fairly strong support for our model, in that it seems to explain the most information in the simplest way.
Our model is also in line with current frontier research that indicates that GD is partly caused by genetic factors and partly by environmental factors. The American College of Paediatricians recently presented a comprehensive position paper on GD in children, which included a detailed summary of the assorted lines of inquiry with regard to the causes of GD more generally. They conclude that GD is for the largest part caused by environmental factors, while acknowledging that twin studies have found a modest genetic influence, with estimates ranging from 20 to 28\% (Diamond, 2013). However, some studies estimate a genetic influence of roughly $30 \%$ in adults (Polderman et al., 2018) or even 50\% in males and 40\% in females (Zucker et al., 2016). It should be noted, however, that according to a systematic literature review by Polderman et al. (2018), the genetic component of gender dysphoria is considerably higher, up to 0.7 , among early-onset GD than among adolescent-onset GD, where the genetic component has been found to be as low as 0.17 (specifically among females with GD). Thus, the common notion that GD reflects being 'born into the wrong body' seems to hold considerable water for early-onset GD but not for adolescent-onset GD. GD is at least as much environmental as it is genetic in males and certainly more so in females.

Pursuing the environmental dimension of GD further, our model also helps to make sense of some of the observations documented in clinical studies on people with GD. Boys who later experience GD tend to have been raised in suboptimal environments, in which the parents frequently fight and in which the fathers have low self-esteem and are distant, while the mothers are high on psychopathology (including traits such as aggressiveness). According to Zucker et al. (2003), these factors increase the risk that boys develop an unclear sense of self-value and a high level of anxiety. Zucker et al. (2003) suggest that these conditions may result in sexual identity problems, and that GD thus has a significant environmental element. However, parental aggressiveness might itself be partly underpinned by elevated androgens and this is itself both heritable and associated with ASD. This would, therefore, connect these environmental variables to our model because they elevate the development of an unclear or inaccurate sense of self. Still, even these associations may to a greater or lesser extent be explained by confounding genetic influences as per usual with family environments, where all individuals are genetically related.

Clinical observation of girls with GD indicates that they tend to have distant mothers with whom they are often in conflict, and fathers who abuse their mothers, possibly leading the girl to identify as a boy (Zucker et al., 2003). Clinical observation studies suffer from a range of problems and may be criticised for small sample sizes (e.g. Wu, 2016), but it must also be recognised that they are generally the only studies available when exploring rare disorders. As our model would predict, from the expectation that all dysphoria have similar 
underpinnings, there is evidence that girls with anorexia tend to have emotionally distant mothers. Their mothers are more likely to suffer from alexithymia, that is, having difficulties verbalising and otherwise expressing emotions (Balottin et al., 2014). Females with GD tend not only merely to exhibit ASD symptoms but also evidence being higher on measures of psychopathology (Kaltiala-Heino et al., 2015). The evidence of an association between psychopathology and GD can be argued to further substantiate, albeit indirectly, the hypothesis that GD is a reflection of masculinisation. Psychopathic traits are also associated with higher levels of masculinisation (Baron-Cohen, 2002) as they include 'male' traits such as low empathy. If present in people with GD, psychopathic traits are very likely to have been inherited from parents, as they are heritable at about 0.5 (Blonigen et al., 2005). This adds support to the hypothesis that masculinisation may be relevant for explaining some of the factors underlying GD at the genetic level. Finally, we did not consider associations between GD and personality disorders, but that would be another important area in which to test our model. We would expect a greater incidence of personality disorders among GD persons, but that is trivial as it might result either from negative experiences due to having GD or from common causes associated with androgen exposure. More specifically would our model predict a stronger incidence among transwomen than among transmen, as it indicates a common role for androgens. Both these effects were found in a comparison of GD and non-GD persons (Duisin et al., 2014).

At large, our model is consistent with the evidence accrued from these clinical studies. It is appreciated, however, that these are small studies and that there may exist different but yet unpublished observations. If so, their publication would be an important contribution to understanding this area in greater depth.

Our findings, however, raise the question why elevated masculinisation should be associated with GD and, indeed, with other dysphoria? By what mechanisms does this relationship occur? We have already noted the observation by Zucker et al. (2003) that boys with GD appear to suffer from high anxiety. This would be consistent with their elevated prevalence of ASD. People with Asperger's syndrome and other ASD traits tend to experience elevated anxiety. ASD is associated with social anxiety among individuals in the normal IQ range and also with general anxiety and with depression (Strang et al., 2013). Among an ASD sample aged 6-18, $40 \%$ had borderline or clinical depression or anxiety while $30 \%$ were firmly within the clinical range, as compared with $6 \%$ in the general population (Strang et al., 2013).

The reasons that people with ASD suffer from anxiety was explored in some detail by Wood and Gadow (2010), who concluded that anxiety has a symbiotic relationship with ASD. One the one hand, it is a 'downstream consequence' of ASD symptoms, such as stress brought about by social rejection, and, on the other hand anxiety will make the ASD symptoms even worse, as 'social skill deficits and repetitive behaviours may be exacerbated by anxiety'. There are also indications that ASD sufferers are more likely than neuro-typical people to lack a clear sense of self. They lack an understanding of who they are, as they fail to comprehend how others perceive them (e.g. Waltz, 2000; Harris \& Leevers, 2000). The brains of ASD sufferers have been found to exhibit reduced connectivity in cortical areas that relate to understanding facial expressions and to theory of mind, and also to 'sense of self' (Cheng et al., 2015). A brain imaging study found that the part of the brain involved in sense of self operates very differently in autistic persons, in that its activity does not reduce during cognitive tasks. It was thus concluded that 'a core deficit in autism might be related to the construal of a sense of self in its relationship with others' (Iacoboni, 2006). On this basis, then, we would expect those with ASD to not only be relatively high in anxiety but to also be confused about or unhappy about who they are. Accordingly, it would follow that we might expect to see GD, to an elevated degree, among ASD sufferers. Indeed, the American College of Pediatricians (2018) has concluded that GD is causally related to anxiety and depression. They aver that those who have been raised in an unstable and conflict-ridden environment may suffer from GD as an indirect result. In such an unstable environment they are also less likely, argue the College, to develop a strong sense of self.

This raises a second question, which is: Which model of GD best explains our findings and the association with ASD and anxiety outlined above? Based on our findings, the genetic/hormonal model does not appear to explain adolescent-onset GD, while it may explain early-onset GD. This conclusion is indeed based on all the predictions that the competing models explicitly or implicitly generated. The support for an association between GD and androgen exposure that we have found would appear to make particular sense viewed through the model propounded by Blanchard (1993). Blanchard argues that some transwomen are 'homosexual transsexuals' who are highly feminised and want to become, as far as possible, heterosexual women. They show signs of opposite-sex behaviour at very young ages. We suggest that it this kind of GD is most parsimoniously explained by the genetic/hormonal model examined above. The rest are what he calls 'autogynephilic transsexuals'. These are male fetishists, argues Blanchard, who are deeply emotionally satisfied by the idea of having a female body, something which correlates with wanting to take action to obtain one, and which becomes an interest for such people during or after adolescence. More recently, Blanchard has averred that his model is also likely to apply to transmen (Bailey \& Blanchard, 2017). If he is right, this would be consistent with our hypothesis that transmen display pronounced masculinity, to the extent of often being lesbians after their transition. The advantage of Blanchard's so-called transsexualism typology is that it provides a plausible model with regard to how those with ASD, whom we would expect to display masculine traits, can reach 
a situation, via their high anxiety, where they may wish to undergo a sex change. It does this by distinguishing between two kinds of GD and conceiving of the more common kind as, in effect, a fetish. We appreciate that this word has sexual and prurient connotations, but this is the term Blanchard employs. Following Blanchard's model, those who are high in ASD are high in androgens, leading to masculinised behaviours and features. However, the ASD also leads to anxiety, which results in fetishes, as those are, according to Blanchard, associated. For discussions of the association between anxiety, trauma and fetishes, see Kafka (2010).

We appreciate that more research is needed to evaluate our model, and that Blanchard's model has been criticised for drawing upon studies with small samples (e.g. Wu, 2016). Also, some more specific aspects of his original study, with regard to subtypes within the homosexual transsexual category, have not been found to replicate (Nuttbrock et al., 2011). Whether or not he has managed to prove the veracity of his own model is beyond the scope of our inquiry. What we can say is that his general model would appear to parsimoniously make sense of the pattern of results that we have found. We also appreciate that this a sensitive issue and that transgender persons may find it upsetting for their condition to be explored in terms of environmental causes. Indeed, they may aver that they were simply born into the wrong body and it took them a while to realise this, and this may well be correct. However, it is important to pursue the theoretical development of the aetiology of GD, in order to eventually reduce the suffering that this condition entails.

Our cautious conclusion is that GD is a manifestation of masculinisation and that, due to the conditions this can lead to, this can result in males identifying as female or females identifying as males. A severe limitation is that this condition and its causes are poorly understood. The concepts of 'dysphoria' and 'paraphilia' are distinct theoretical categories which appear to cross-over, and future research can hopefully hone these categories of analysis. It would be useful - to the extent it is possible - to explore the correlates of other even more unusual dysphoria, such as transracialism. In terms of social and policy implications, our conclusion, as well as the fact that there are several different forms of GD, points to the importance of carefully assessing the causes of the GD condition in each individual, so that the best set of interventions can be deployed. It also suggests that irreversible treatments should be very restrictively employed, as they may not resolve the underlying cause of the experienced problems, and may, instead, lead to further problems for these individuals.

Acknowledgements Open access funding provided by Umea University.

\section{Compliance with Ethical Standards}

Conflict of Interest The authors declare that they have no conflict of interest.
Open Access This article is licensed under a Creative Commons Attribution 4.0 International License, which permits use, sharing, adaptation, distribution and reproduction in any medium or format, as long as you give appropriate credit to the original author(s) and the source, provide a link to the Creative Commons licence, and indicate if changes were made. The images or other third party material in this article are included in the article's Creative Commons licence, unless indicated otherwise in a credit line to the material. If material is not included in the article's Creative Commons licence and your intended use is not permitted by statutory regulation or exceeds the permitted use, you will need to obtain permission directly from the copyright holder. To view a copy of this licence, visit http://creativecommons.org/licenses/by/4.0/.

\section{References}

American College of Pediatricians. (2018). Gender dysphoria in children. Retrieved: https://www.acpeds.org/the-college-speaks/positionstatements/gender-dysphoria-in-children.

American Psychiatric Association. (1980). Diagnostic and statistical manual of mental disorders (3rd ed.). Washington, DC: American Psychiatric Association.

American Psychiatric Association. (2013). Diagnostic and statistical manual of mental disorders (5th ed.). Arlington, VA: American Psychiatric Publishing.

Atkinson, B., Smulders, T., \& Wallenberg, J. (2017). An endocrine basis for tomboy identity: The second-to-fourth digit ratio (2D:4D) in "tomboys". Psychoneuroendocrinology, 79, 9-12.

Auyeung, B., Taylor, K., Hackett, G., \& Baron-Cohen, S. (2010). Foetal testosterone and autistic traits in 18 to 24 -month-old children. Molecular Autism, 1, 11.

Bailey, J. M. \& Blanchard, R. (2017). Gender dysphoria is not one thing. 4thWaveNow. Retrieved: https://4thwavenow.com/tag/ autohomoerotic-gender-dysphoria/.

Bale, T. L., \& Epperson, C. N. (2017). Sex as a biological variable: Who, what, when, why, and how. Neuropsychopharmacology, 42, 386396.

Balottin, L., Nacinovich, R., Bomba, M., \& Mannarini, S. (2014). Alexithymia in parents and adolescent anorexic daughters: Comparing the responses to TSIA and TAS-20 scales. Neuropsychiatric Disease and Treatment, 10, 1941-1951.

Balthazart, J. (2011). Mini-review: Hormones and human sexual orientation. Endocrinology, 152, 2937-2947.

Baron-Cohen, S. (2002). The extreme male brain theory of autism. Trends in Cognitive Sciences, 6, 248-254.

Bejerot, S., \& Eriksson, J. (2014). Sexuality and gender role in autism spectrum disorder: A case control study. PLOS One. https://doi.org/ 10.1371/journal.pone.0087961.

Berenbaum, S. A., \& Beltz, A. M. (2016). How early hormones shape gender development. Current Opinion in Behavioral Sciences, 7 , 53-60.

Blanchard, R. (1993). Varieties of autogynephilia and their relationship to gender dysphoria. Archives of Sexual Behavior, 22, 241-251.

Blanchard, R., Clemmensen, L., \& Steiner, B. (1987). Heterosexual and homosexual gender dysphoria. Archives of Sexual Behavior, 16, $139-152$.

Blanchard, R., \& Lippa, R. (2007). Birth order, sibling sex ratio, handedness, and sexual orientation of male and female participants in a BBC Internet research project. Archives of Sexual Behavior, 36, 163-176.

Blonigen, D. M., Hicks, B. M., Krueger, R. F., Patrick, C. J., \& Iacono, W. G. (2005). Psychopathic personality traits: Heritability and 
genetic overlap with internalizing and externalizing psychopathology. Psychological Medicine, 35, 637-648.

Burgaleta, M., Head, K., Alvarez-Linera, J., Martinez, K., Escorial, S., Haier, R., \& Colom, R. (2012). Sex differences in brain volume are related to specific skills, not to general intelligence. Intelligence, 40 , 60-68.

Buss, D. (1989). The evolution of desire: Strategies of human mating. New York: Basic Books.

Cahill, L. (2006). Why sex matters for neuroscience. Nature Reviews Neuroscience, 7, 477-484.

Cheng, W., Rolls, E., Gu, H., Zhang, J., \& Geng, J. (2015). Autism: Reduced connectivity between cortical areas involved in face expression, theory of mind, and the sense of self. Brain, 138, 13821393.

Committee on Understanding Premature Birth. (2007). Preterm birth: Causes, consequences and prevention. Washington, DC: National Academies Press.

Danker, S., Narayan, S. K., Bluebond-Langner, R., Schechter, L. S., \& Berli, J. U. (2018). A survey study of surgeons' experience with regret and/or reversal of gender-confirmation surgeries. Plastic and Reconstructive Surgery - Global Open, 6, 189. https://doi.org/ 10.1097/01.GOX.0000547077.23299.00.

Datta Banik, S. (2017). Handedness and bilateral asymmetry of second to fourth finger digit ratio (2D:4D) in adults from Merida, Mexico. Human Biology Review, 6, 79-95.

Dewinter, J., Vermeiren, R., Vanwesenbeek, I., \& Van Nieuwenhuizen, C. (2013). Autism and normative sexual development: A narrative review. Journal of Clinical Nursing, 22, 3467-3483.

Diamond, M. (2013). Transsexuality among twins: Identity concordance, transition, rearing, and orientation. International Journal of Transgenderism, 14, 24-38.

Duisin, D., Batinic, B., Barisic, J., Djordevic, M. L., Vuovic, S., \& Bisic, M. (2014). Personality disorders in persons with gender identity disorder. Scientific World Journal, 2014, 809058-809057. https:// doi.org/10.1155/2014/809058.

Ellis, L., Skorska, M., \& Bogaert, A. (2017). Handedness, sexual orientation, and somatic markers for prenatal androgens: Are southpaws really that gay? Laterality, 22, 157-180.

Fraser, L. (2015). Gender dysphoria: Definition and evolution through the years (pp. 19-31). In C. Trombetta, G. Liguori, \& M. Bertolotto (Eds.), Management of gender dysphoria: A multidisciplinary approach. Springer-Verlag Italia: Milan.

Geary, D. C. (2017). Evolutionary framework for identifying sex- and species-specific vulnerabilities in brain development and functions. Journal of Neuroscience Research, 95, 355-361.

Gillberg, C. (1983). Autistic children's hand preferences: Results from an epidemiological study of infantile autism. Psychiatry Research, 10, 21-30.

Glidden, D., Bouman, W., Jones, B., \& Arcelus, J. (2016). Gender dysphoria and autism spectrum disorder: A systematic review of the literature. Sexual Medicine Reviews, 4, 3-14

Goodman, M., Adams, N., Cornell, T., Kreukels, B., Motmans, J., \& Coleman, E. (2019). Size and distribution of transgender and gender nonconforming populations: A narrative review. Endocrinology and Metabolism Clinics of North America, 48, 303-321.

Green, R., \& Young, R. (2001). Hand preference, sexual preference and transsexualism. Archives of Sexual Behavior, 30, 565-574.

Greenberg, D., Warrier, V., Allison, C., \& Baron-Cohen, S. (2018). Testing the empathizing-systemizing theory of sex differences and the extreme male brain theory of autism in half a million people. PNAS, 115, 12152-12157. https://doi.org/10.1073/pnas. 1811032115 .

Gur, R. C., \& Gur, R. E. (2017). Complementarity of sex differences in brain and behaviour: From laterality to multimodal neuroimaging. Journal of Neuroscience Research, 95, 189-199.
Hare, L., Bernard, P., Sánchez, F. J., Baird, P. N., Vilain, E., Kennedy, T., \& Harley, V. R. (2009). Androgen receptor repeat length polymorphism associated with male-to-female transsexualism. Biological Psychiatry, 65, 93-96.

Harris, P.-L., \& Leevers, H.-J. (2000). Pretending, imagery and selfawareness in autism. In S. Baron-Cohen, H. Tager-Flusberg, \& D.-J. Cohen (Eds.), Understanding other minds: Perspectives from autism and cognitive neuroscience. Oxford: Oxford University Press.

Hausmann, M. (2017). Why sex hormones matter for neuroscience: A very short review on sex, sex hormones, and functional brain asymmetries. Journal of Neuroscience Research, 95, 40-49.

Heylens, G., Aspeslagh, L., Dierickx, J., Baetens, K., Van Hoorde, B., De Cuypere, G., \& Elaut, E. (2018). The co-occurrence of gender dysphoria and autism spectrum disorder in adults: An analysis of crosssectional and clinical chart data. Journal of Autism and Developmental Disorders, 48, 2217-2223.

Hirnstein, M., Hugdahl, K., \& Hausmann, M. (2019). Cognitive sex differences and hemispheric asymmetry: A critical review of 40 years of research. Laterality: Asymmetries of Body, Brain and Cognition, 24, 204-252.

Hisasue, S.-I., Sasaki, S., Tsukamoto, T., \& Horie, S. (2012). The relationship between second-to-fourth digit ratio and female gender identity. Journal of Sexual Medicine, 9, 2903-2910.

Hönekopp, J., \& Watson, S. (2010). Meta-analysis of digit ratio 2D:4D shows greater sex difference in the right hand. American Journal of Human Biology, 22, 619-630.

Houssayni, S., \& Nilsen, K. (2018). Transgender competent provider: Identifying transgender health needs, health disparities, and health coverage. Kansas Journal of Medicine, 11, 15-19.

Iacoboni, M. (2006). Failure to deactivate in autism: The co-constitution of self and other. Trends in Cognitive Sciences, 10, 431-433.

Jahanshad, N., \& Thompson, P. (2017). Multimodal neuroimaging of male and female brain structure in health and disease across the life span. Journal of Neuroscience Research, 95, 371-379.

James, S. E., Herman, J. L., Rankin, S., Keisling, M., Mottet, L., \& Anafi, M. (2016). The report of the 2015 U.S. transgender survey. Washington, DC: National Center for Transgender Equality.

Jeffrey, A., Shackelford, T., Zeigler-Hill, V., Vonk, J., \& McDonald, M. (2019). The evolution of human female sexual orientation. Evolutionary Psychological Science, 5, 71-86.

Jones, R., Wheelwright, S., Farrell, K., Martin, E., Green, R., Di Ceglie, D., \& Baron-Cohen, S. (2012). Brief report: Female-to-male transsexual people and autistic traits. Journal of Autism and Developmental Disorders, 41, 301-306.

Kafka, M. (2010). The DSM diagnostic criteria for fetishism. Archives of Sexual Behavior, 39, 357-362.

Kaltiala-Heino, R., Sumia, M., Työläjärvi, M., Lindberg, N., et al. (2015). Two years of gender identity service for minors: Overrepresentation of natal girls with severe problems in adolescent development. Child and Adolescent Psychiatry and Mental Health, 9, 9.

Kaltiala-Heino, R., Työläjärvi, M. \& Lindberg, N. (2019). Gender dysphoria in adolescent population: A 5-year replication study. Clinical Child Psychology and Psychiatry, 24, 379-387 doi.org/https://doi. org/10.1177/1359104519838593.

Khahil, R., \& Richa, S. (2012). Apotemnophilia or body integrity identity disorder: A case report review. International Journal of Lower Extremity Wounds, 11, 313-319.

Kobylynska, L., Anghel, C., Mihailescu, I., Rad, F., \& Dobrescu, I. (2017). Handedness in children with autism spectrum disorders. European Psychiatry, 41(S), 214.

Kraemer, B., Noll, T., Delsignore, A., Milos, G., Schnyder, U., \& Hepp, U. (2009). Finger length ratio (2D:4D) in adults with gender identity disorder. Archives of Sexual Behavior, 38, 359-363.

Lai, M.-C., Lerch, J., Floris, D., Ruigrok, A. N. V., Pohl, A., Lombardo, M. V., \& Baron-Cohen, S. (2017). Imaging sex/gender and autism 
in the brain: Etiological implications. Journal of Neuroscience Research, 95, 380-397.

Lalumiere, M. L., Blanchard, R., \& Zucker, K. J. (2000). Sexual orientation and handedness in men and women: A meta-analysis. Psychological Bulletin, 126, 575-592.

Leinung, M., \& Wu, C. (2017). The biologic basis of transgender identity: 2D:4D finger length ratios implicate a role for prenatal androgen activity. Endocrine Practice, 23, 669-671.

Lippa, R. (2010). Gender differences in personality and interests: When, where, and why? Social and Personality Psychology Compass, 4, $1098-1110$.

Littman, L. (2018). Parent reports of adolescents and young adults perceived to show signs of a rapid onset of gender dysphoria. PLoS One, 13(8), e0202330. https://doi.org/10.1371/journal.pone. 0202330 .

Luders, E., Sanchez, F. J., Gaser, C., et al. (2009). Regional gray matter variation in male-to-female transsexualism. Neuroimage, 46, 904 907.

Manning, J., Baron-Cohen, S., Wheelwright, S., \& Sanders, G. (2001). The 2 nd to 4 th digit ratio and autism. Developmental Medicine and Child Neurology, 43, 160-164.

Manning, J., Trivers, R., \& Fink, B. (2017). Is digit ratio (2D:4D) related to masculinity and femininity? Evidence from the BBC Internet study. Evolutionary Psychological Science, 3, 316-324.

Miller, C. (2015). The search for the best estimate of the transgender population. New York Times, https://www.nytimes.com/2015/06/ 09/upshot/the-search-for-the-best-estimate-of-the-transgenderpopulation.html.

Miller, E. M. (2000). Homosexuality, birth order, and evolution: Toward an equilibrium reproductive economics of homosexuality. Archives of Sexual Behavior, 29, 1-34.

Mustanski, B., Bailey, J. M., \& Kaspar, S. (2002). Dermatoglyphics, handedness, sex, and sexual orientation. Archives of Sexual Behavior, 31, 113-122.

Nadler, A., Carmerer, C., Zava, D., et al. (2019). Does testosterone impair men's cognitive empathy? Evidence from two large-scale randomized controlled trials. Proceedings of the Royal Society B, 286(1910), 20191062. https://doi.org/10.1098/rspb.2019.1062.

Nuttbrock, L., Bokting, W., Mason, M., et al. (2011). A further assessment of Blanchard's typology of homosexual versus nonhomosexual or autogynephilic gender dysphoria. Archives of Sexual Behavior, 40, 247-257.

Office for National Statistics. (2016). Sexual identity, UK: 2016. R e tri e ved: ht t p s : / / w w w o n s.g o v. u k/ peoplepopulationandcommunity/culturalidentity/sexuality/ bulletins/sexualidentityuk/2016.

Øien, R., Cichetti, D., \& Nordahl-Hansen, A. (2018). Gender dysphoria, sexuality and autism spectrum disorders: A systematic map review. Journal of Autism and Developmental Disorders, 48, 4028-4037.

Orlebeke, J. F., Boomsma, D. I., Gooren, L. J., Verschoor, A. M., \& Van Den Bree, M. J. M. (1992). Elevated sinistrality in transsexuals. Neuropsychology, 6, 351-355.

Papadatou-Pastou, M., Martin, M., Munafò, M., \& Jones, G. (2008). Sex differences in left-handedness: A meta-analysis of 144 studies. Psychological Bulletin, 134, 677-699.

Parekh, R. (2016). What is gender dysphoria? American Psychiatric Association. https://www.psychiatry.org/patients-families/genderdysphoria/what-is-gender-dysphoria.

Pertoldi, C., Kristensen, T., Anderson, D., \& Loeschcke, V. (2006). Developmental instability as an estimator of genetic stress. Heredity, 96, 122-127.

Polderman, T., Kreukels, B., Irwing, M., et al. (2018). The biological contributions to gender identity and gender diversity: Bringing data to the table. Behavior Genetics, 48, 95-108.
Quinton, S., Smith, A., \& Joiner, T. (2011). The 2nd to 4th digit ratio (2D: 4D) and eating disorder diagnosis in women. Personality and Individual Differences, 51, 402-405.

Ribeiro, E., Neave, N., Morais, R., \& Manning, J. (2016). Direct versus indirect measurement of digit ratio (2D:4D): A critical review of the literature and new data. Evolutionary Psychology, 14, 1474704916632536.

Ridley, R. (2019). Some difficulties behind the concept of the 'extreme male brain' in autism research. A theoretical review. Research in Autism Spectrum Disorders, 57, 19-27.

Ristori, J., Fisher, A., Castellini, G., et al. (2019). Gender dysphoria and anorexia nervosa symptoms in two adolescents. Archives of Sexual Behavior, 48, 1625-1631.

Ruta, L., Ingudomnukul, E., Taylor, K., Chakrabarti, B., \& Baron-Cohen, S. (2011). Increased serum androstenedione in adults with autism spectrum conditions. Psychoneuroendocrinology, 36, 1154-1163.

Soper, H., Satz, P., Orsini, D., Henry, R., Zvi, J., \& Schulman, M. (1986). Handedness patterns in autism suggests subtypes. Journal of Autism and Developmental Disorders, 16, 155-167.

Sparks, B., Friedman, S., Shaw, D., et al. (2002). Brain structural abnormalities in young children with autism spectrum disorder. Neurology, 59, 2. https://doi.org/10.1212/WNL.59.2.184.

Sperling, W., Biermann, T., Bleich, S., et al. (2010). Non-righthandedness and free serum testosterone levels in detoxified patients with alcohol dependence. Alcohol and Alcoholism, 3, 237-240.

Stagg, S., \& Vincent, J. (2019). Autistic traits in individuals self-defining as transgender or nonbinary. European Psychiatry, 61, 17-22.

Strang, J., Kenworthy, L., Daniolos, P., et al. (2013). Depression and anxiety symptoms in children and adolescents with autism spectrum disorders without intellectual disability. Research in Autism Spectrum Disorders, 6, 406-412.

Su, R., Rounds, J., \& Armstrong, P. I. (2009). Men and things, women and people: A meta-analysis of sex differences in interests. Psychological Bulletin, 135, 859-884.

Swift-Gallant, A., Johnson, B., Di Rita, V., \& Breedlove, S. M. (2020). Through a glass, darkly: Human digit ratios reflect prenatal androgens, imperfectly. Hormones and Behavior, 120, 104686.

Tenconi, E., Santonastaso, P., Degortes, D., Bosello, R., Titton, F., Mapelli, D., \& Favaro, A. (2010). Set-shifting abilities, central coherence, and handedness in anorexia nervosa patients, their unaffected siblings and healthy controls: Exploring putative endophenotypes. World Journal of Biological Psychiatry, 11, 813-823.

Turban, J., \& Keuroghlian, A. (2018). Dynamic gender presentations: Understanding transition and de-transition among transgender youth. Journal of the American Academy of Child and Adolescent Psychiatry, 57, 451-453.

Turban, J., \& Van Schalkwyk, G. (2018). "Gender dysphoria" and autism spectrum disorder: Is the link real? Journal of the American Academy of Child and Adolescent Psychiatry, 57, 8-9.e2. https:// doi.org/10.1016/j.jaac.2017.08.017.

Van der Linden, D., Dunkel, C., \& Madison, G. (2017). Sex differences in brain size and general intelligence $(g)$. Intelligence, 63, 78-88.

Van der Miesen, A., De Vries, A., Steensma, T., \& Hartman, C. (2018). Autistic symptoms in children and adolescents with gender dysphoria. Journal of Autism and Developmental Disorders, 48, 15371548

Van der Miesen, A. I., Hurley, H., \& De Vries, A. L. (2016). Gender dysphoria and autism spectrum disorder: A narrative review. International Review of Psychiatry, 28, 70-80.

Voracek, M., Kaden, A., Kossmeier, M., Pietschnig, J., \& Tran, U. S. (2018). Meta-analysis shows associations of digit ratio (2D:4D) and transgender identity are small at best. Endocrine Practice, 24, 386390. 
Vujović, S., Popović, S., Marojević, L. et al. (2014). Finger length ratios in Serbian transsexuals. The Scientific World Journal. Retrieved: https://doi.org/10.1155/2014/763563

Wallien, M., Zucker, K., Steensma, T., \& Cohen-Kettenis, P. (2008). 2D: 4D finger-length ratios in children and adults with gender identity disorder. Hormones and Behavior, 54, 450-454.

Waltz, M. (2000). Obsessive compulsive disorder. New York: O'Reilly.

Watson, D., \& Coren, S. (1992). Left-handedness in male-to-female transsexuals. JAMA, 267, 1342.

Westwood, H., \& Tchanturia, K. (2017). Autism Spectrum disorder in anorexia nervosa: An updated literature review. Current Psychiatry Reports, 19, 41.

Wilson, E. O. (1998). Consilience: The Unity of knowledge. New York: Knopf.

Wood, J., \& Gadow, K. (2010). Exploring the nature and function of anxiety in youth with autism spectrum disorders. Clinical Psychology: Science and Practice, 17, 281-292.

Woodley of Menie, M. A., Fernandes, H., Kanazawa, S., \& Dutton, E. (2018). Sinistrality is associated with (slightly) lower general intelligence: A data synthesis and consideration of secular trend data in handedness. HOMO: Journal of Comparative Human Biology, 69, 118-126.

Wu, K. J. (2016). Between the (gender) lines: The science of transgender identity. Science in the News, http://sitn.hms.harvard.edu/flash/ 2016/gender-lines-science-transgender-identity/.

Zucker, K., Beaulieu, N., Bradley, S., Grimshaw, G., \& Wilcox, A. (2001). Handedness in boys with gender identity disorder. Journal of Child Psychology and Psychiatry, 42, 767-776.

Zucker, K., Bradley, S., Ben-Dat, D., et al. (2003). Psychopathology in the parents of boys with gender identity disorder. Journal of the American Academy of Child Adolescent Psychiatry, 42, 2-4.

Zucker, K., Lawrence, A., \& Kreukels, B. (2016). Gender dysphoria in adults. Annual Review of Clinical Psychology, 12, 217-247.

Publisher's Note Springer Nature remains neutral with regard to jurisdictional claims in published maps and institutional affiliations. 